\title{
Drinking water source and human Toxoplasma gondii infection in the United States: a cross-sectional analysis of NHANES data
}

\author{
Whitney S Krueger ${ }^{1,2}$, Elizabeth D Hilborn ${ }^{2}$, Reagan R Converse ${ }^{1,2}$ and Timothy J Wade ${ }^{2^{*}}$
}

\begin{abstract}
Background: Toxoplasma gondii imparts a considerable burden to public health. Human toxoplasmosis can be life-threatening in immunocompromised individuals, has been associated with psychiatric disorders, and can cause severe congenital pathologies, spontaneous abortion, or stillbirth. Environmental modes of transmission contributing to the incidence of human toxoplasmosis are poorly understood. We sought to examine National Health and Nutrition Examination Survey (NHANES) data for risk factors associated with T. gondii seroprevalence.

Methods: T. gondii serology results reported for Continuous NHANES survey years 1999-2004 and 2009-10 were examined. To explore associations with toxoplasmosis seropositivity, covariates of interest were selected a priori, including source and home treatment of tap water. Associations between potential risk factors and evidence of lgG antibodies against $T$. gondii were assessed using multivariable logistic regression.
\end{abstract}

Results: Among 23,030 participants with available T. gondii serology across 8 years of continuous NHANES survey data (1999-2004; 2009-2010), persons born outside the United States were significantly more likely to be seropositive, and seropositivity was inversely associated with years spent in the United States. Among US-born participants, participants with homes on well water (both those who used at-home water treatment devices and those who did not), as well as participants with public/private company-provided tap water who did not use at-home water treatment devices, were significantly more likely to be seropositive compared to participants who used home treatment devices on tap water provided by a private or public water company. A comparative subpopulation analysis revealed age-adjusted seroprevalence among US-born persons $12-49$ yrs old significantly declined to $6.6 \%(95 \% \mathrm{Cl}, 5.2-8.0)(P<0.0001)$ in 2009-10, compared to previously published reports for NHANES data from 1988-1994 (14.1\%) and 1999-2004 (9.0\%).

Conclusions: Data suggests that T. gondii infections continue to decline in the United States, but the overall infection rate remains substantial at nearly $7 \%$. Despite the limitations in the Continuous NHANES cross-sectional survey, the association between well water use and T. gondii infection warrants further research.

Keywords: Nutrition surveys, Toxoplasma, Drinking water, Water wells, Cross-sectional studies

\section{Background}

Toxoplasma gondii, an obligate intracellular protozoan, is one of the most widespread zoonotic parasites in the world. T. gondii infects all warm-blooded animals, but utilizes domestic and wild cats in the Felidae family as its definitive host [1-3]. T. gondii infects humans via ingestion of undercooked meat containing trophozoites or

\footnotetext{
* Correspondence: wade.tim@epa.gov

Environmental Public Health Division, Office of Research and Development, US Environmental Protection Agency, 104 Mason Farm Road, Chapel Hill, North Carolina, USA

Full list of author information is available at the end of the article
}

tissue cysts, or through ingestion of sporulated oocysts found in food, soil, and water recently contaminated with cat feces $[1,4,5]$. When oocysts are ingested by an intermediate host such as humans [6], they migrate in the host's body and form permanent cysts in various organs and tissues, but most commonly in the brain [7]. Toxoplasmosis is a lifelong infection that has the potential to reactivate and cause severe disease and even death in immunocompromised individuals.

Though primary toxoplasmosis is usually subclinical, sequelae can range from mild flu-like symptoms to seizures 
and confusion. Severity of symptoms may depend upon the parasite stage ingested, with infections from oocysts believed to elicit more clinically severe manifestations than ingestion of tissue cysts [4]. Immunocompromised individuals can experience more severe symptoms, either during primary infection or following reactivation of latent cysts $[8,9]$. Likely attributed to cysts in the brain, psychiatric disorders including mood disorders, personality changes, and schizophrenia have been associated with chronic $T$. gondii infections; however, the underlying etiology is not entirely clear $[10,11]$. Of special public health concern, fetuses can be infected via vertical transmission when a woman experiences a primary infection during pregnancy. Disseminating tachyzoites colonize placental tissues and infect the fetus, potentially causing severe injury, stillbirth, or spontaneous abortion. Congenital toxoplasmosis results in the highest burden of disease for human $T$. gondii infections [6].

T. gondii has infected an estimated $25-30 \%$ of the world's population [9]; however prevalence varies across countries/geographical regions [12]. North America has a lower prevalence (10-30\%) compared to Latin America and tropical regions of Africa, which have a $50-80 \%$ prevalence. In addition to felid presence and density, climatic, anthropogenic, economic, social, and cultural factors (e.g. hygienic practices, dietary habits, cooking methods), as well as water quality and sanitation coverage all play important roles in T. gondii environmental persistence, zoonotic transmission, and prevalence in humans [1]. In the United States, toxoplasmosis is responsible for one of the highest direct costs to health care among diseases partially associated with water-borne transmission [13].

Because recent epidemiological studies could not attribute the majority of $T$. gondii infections to contaminated food consumption or soil-related exposures [14,15], other sources of contamination are likely starting to play a key role in transmitting $T$. gondii to humans, especially in the United States [16]. In the environment, oocysts can resist freezing and moderately high temperatures to remain viable for years in soil and water [4,17]. Water chemical and physical treatments, including chlorination and ozone treatment, are not effective in killing oocysts; however, oocysts are relatively susceptible to ultraviolet radiation $[18,19]$. Although modern municipal water treatment systems (with filtering, coagulation, flocculation, and settling) are generally effective at removing oocysts [4], many populations do not have access to drinking water that has undergone this level of treatment. Drinking water is an endemic source of T. gondii in Brazil and Guatemala, and drinking water has been implicated as the source of toxoplasmosis outbreaks in several countries [4], including Panama [20] and Canada [21,22]. Oocysts have not been directly detected in US drinking water, but assessing environmental contamination of $T$. gondii is technically difficult
[23]. Researchers have hypothesized that ingesting inadequately treated drinking water may be a significant source for human infections [4,24].

The National Health and Nutrition Examination Survey (NHANES), within the National Center for Health Statistics of the Centers for Disease Control and Prevention, has conducted large scale surveys designed to assess changes over time of the health and nutritional status of adults and children in the United States. Descriptive epidemiology reports have previously reported $T$. gondii seroprevalence trends for past NHANES cohorts (1988-1994 and 19992004) [25-28]. Increased odds of seropositivity were reported with increasing age, poverty, foreign country of birth, lower level of education, living in crowded conditions, and working in soil-related occupations, which varied by race/ethnicity [25]. To assess the disease burden in the United States, a comparative subpopulation analysis of US-born adults aged 12-49 years revealed that $T$. gondii seroprevalence declined from $14.1 \%$ during the Third NHANES (1988-1994) to 9.0\% during the Continuous NHANES cycles from 1999-2004 [27].

Because the CDC recently released seroprevalence data for $T$. gondii among eligible participants in the Continuous NHANES 2009-10 cycle, we examined all available Continuous NHANES data (1999-2004 and 2009-10) to expand on the previous literature and look for evidence of environmental risk factors associated with $T$. gondii seroprevalence among a nationally representative population [3].

\section{Methods}

Four 2-year cycles of Continuous NHANES data (19992000, 2001-02, 2003-04, and 2009-10) were examined [3]. Available serum from eligible participants 6-49 yrs old (1999-2004) and $\geq 6$ yrs old (2009-2010) were tested by the CDC for immunoglobulin G (IgG) antibodies against Toxoplasma gondii by an enzyme immunoassay (EIA). A dichotomous seropositivity cut-off was provided by the CDC (Continuous NHANES 19992004: IgG $\geq 10 \mathrm{IU} / \mathrm{mL}$ = positive; $\mathrm{IgG}<10 \mathrm{IU} / \mathrm{mL}$ = negative; Continuous NHANES 2009-2010: IgG $\geq 33 \mathrm{IU} / \mathrm{mL}=$ positive; IgG $<33 \mathrm{IU} / \mathrm{mL}$ = negative).

Data were collected using a complex survey design; therefore, seroprevalence estimates were weighted to represent the total US civilian non-institutionalized population, and CDC's recommended approaches for SAS survey analysis procedures were followed [3]. All analyses were conducted using SAS v9.3 (Cary, NC). Using all of the available NHANES survey data, the authors a priori selected potential covariates of interest to examine associations with toxoplasmosis seropositivity. Of special interest were blood biomarkers (using complete blood count results) to examine clinical severity and exposure to drinking water, because these factors had not yet been thoroughly 
examined in previous reports. Smoking status was based on serum cotinine level $(\mathrm{ng} / \mathrm{mL})$ to delineate light smokers and second-hand exposure (10-99 $\mathrm{ng} / \mathrm{mL}$ ) from smokers $(\geq 100 \mathrm{ng} / \mathrm{mL})$. The NHANES variable of 'household food security' was determined using the US Food Security Survey Module. Affirmative responses were counted from 18 items for households with children and 10 items for households without children. Categorical data were derived: no affirmative responses $=$ full food security; $1-2=$ marginal food security; 3-5 (without children) or 3-7 (with children) = low food security; 6-10 (without children) and 8-18 (with children) = very low food security. Items included: worried will run out of food, food didn't last, couldn't afford meals, adults cut size or skip meals, eat less than should, hungry but didn't eat, adults don't eat whole day, and use of emergency foods from food banks, soup kitchens, or other organizations. For our analyses, we combined all categories below 'full food security' to create a dichotomous variable.

In the Housing Characteristics (HOQ) questionnaire participants were asked: "What is the source of tap water in this home? Is it a private or public water company, a private or public well, or something else?" and "Are any of the water treatment devices listed on this card used in your home?" Treatment devices listed were: Brita ${ }^{\circ}$ or other pitcher water filter, ceramic or charcoal filter, water softener, aerator, and reverse osmosis. In order to better understand their interaction, the two variables associated with tap water were combined into one covariate with 4 categorical responses (home-treated well water, home-untreated well water, home-treated water company and home-untreated water company).

Seroprevalence of IgG antibodies against $T$. gondii was assessed with multivariable logistic regression to examine associations with key demographic characteristics and potential risk factors associated with seropositivity. Wald chi-square odds ratio (OR) estimates and 95\% confidence intervals (CI) were ascertained for unadjusted and adjusted regression models. Covariates with $\mathrm{p}$ values $<0.1$ were considered for inclusion in multivariable models. Multivariable models were selected using stepwise backwards elimination of covariates with $p$ values $>0.1$. Selected models included all covariates with $\mathrm{p}$ values $<0.05$ as well as any a priori selected demographic characteristics that confounded observed associations between independent variables and the outcome.

\section{Results}

Across the 8 years of Continuous NHANES data for which $T$. gondii seroprevalence was examined (1999-2000, 200102, 2003-04, and 2009-10), T. gondii serology results were available for 23,030 participants (Table 1). Approximately 5,300 participants 6-49 yrs old were included in each of the first 3 Continuous NHANES cycles (1999-2000,
2001-02, and 2003-04). For the more recent cycle (2009$10)$, the eligible population was expanded to all participants $\geq 6$ yrs old; therefore, this cycle had a larger subgroup of over 7,000 participants. T. gondii seroprevalence was also significantly different across key demographic variables, including age, gender, race/ethnicity, and poverty index (Table 1). Controlling for age, there was a statistically significant difference in overall $T$. gondii seroprevalence among the survey years, $(P<0.0001)$ with adjusted odds of seropositivity in both 2001-02 and 2009-10 cycles significantly lower compared to the 1999-2000 cycle (data not shown).

Risk factor associations were first assessed for all 23,030 participants using multivariable logistic regression (Table 2). Increasing age was significantly associated with $T$. gondii seropositivity, and men were more likely to be seropositive than women (adjusted OR [AOR] $=1.2 ; 95 \% \mathrm{CI}, 1.1-1.4$ ). Compared to non-Hispanic Whites, Mexican Americans were less likely to be seropositive (AOR $=0.5$; $95 \% \mathrm{CI}$, 0.40.7), while non-Hispanic Blacks and other Hispanics had significantly elevated adjusted odds of seroprevalence $(\mathrm{AOR}=1.3 ; 95 \% \mathrm{CI}, 1.04-1.6$ and $\mathrm{AOR}=1.6 ; 95 \%$ CI, 1.3-2.1; respectively). Compared to full food security, having marginally secure or insecure household food was associated with higher odds of seropositivity $(\mathrm{AOR}=1.3$; 95\% CI, 1.1-1.5). Smokers had higher odds of seropositivity compared to non-smokers ( $\mathrm{AOR}=1.2 ; 95 \% \mathrm{CI}, 1.03-1.5)$. In addition, the largest effect was observed for country of birth origin. Compared to participants born in the United States, participants born in Mexico had significantly higher odds of seropositivity (AOR $=6.3$; 95\% CI, 4.7-8.4), as did participants born in a foreign country other than Mexico $(\mathrm{AOR}=3.7$; 95\% CI, 3.0-4.6). In examining health correlates, an inverse association was observed with participants' self-reported general health condition - the worse the perceived health condition, the higher odds of seropositivity (Table 2). Participants who reported taking treatment for anemia in the past 3 months were more likely to have antibodies against $T$. gondii $(\mathrm{AOR}=1.4 ; 95 \% \mathrm{CI}$, 1.01-2.0). In unadjusted comparisons there was a significant association between seropositivity and eosinophilia, but the effect diminished in the multivariable model.

Associations between $T$. gondii seroprevalence, tap water source, and using home water treatment devices were observed (Table 2). Compared to participants using hometreated company water, participants with home-untreated well water had a 1.5 times higher adjusted odds (95\% CI, 1.02-2.2) of T. gondii seropositivity. Because there was a high correlation between seropositivity and country of origin, we theorized that if seropositive participants born outside the United States were more likely to be infected while living abroad (where risk of $T$. gondii infection is higher), then their source of drinking water once living in the United States would not have the same association 
Table 1 Toxoplasma gondii seroprevalence ${ }^{a}$ by demographic characteristics for participants $\geq 6$ years old; NHANES 1999-2004, 2009-10

\begin{tabular}{|c|c|c|c|c|}
\hline Variable & $\mathrm{N}$ & 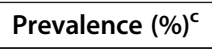 & $95 \% \mathrm{CL}$ & $P$ \\
\hline Overall & 23030 & $2642(11.7)$ & $10.8,12.6$ & \\
\hline Continuous NHANES survey year & & & & 0.0116 \\
\hline $1999-2000$ & 5089 & $602(13.3)$ & $11.6,15.1$ & \\
\hline $2001-2002$ & 5751 & $527(9.3)$ & $7.1,11.4$ & \\
\hline 2003-2004 & 5120 & $434(10.8)$ & $8.6,13.0$ & \\
\hline $2009-2010$ & 7070 & $1079(13.2)$ & $11.9,14.4$ & \\
\hline Age group (years) & & & & $<0.0001$ \\
\hline $6-12$ & 4274 & $155(3.5)$ & $2.5,4.4$ & \\
\hline $13-17$ & 4693 & $279(4.6)$ & $3.7,5.6$ & \\
\hline $18-28$ & 4810 & $488(9.2)$ & $7.7,10.7$ & \\
\hline $29-43$ & 4799 & $757(14.0)$ & $12.4,15.6$ & \\
\hline $44-80$ & 4450 & $963(17.7)$ & $16.0,19.4$ & \\
\hline Gender & & & & 0.0133 \\
\hline Male & 11239 & $1402(12.6)$ & $11.4,13.8$ & \\
\hline Female & 11791 & $1240(10.8)$ & $9.8,11.9$ & \\
\hline Race/ethnicity & & & & $<0.0001$ \\
\hline Non-Hispanic White & 8546 & $854(9.9)$ & $8.8,11.0$ & \\
\hline Non-Hispanic Black & 5535 & $566(12.6)$ & $10.7,14.5$ & \\
\hline Mexican American & 6441 & 749 (13.6) & $11.7,15.5$ & \\
\hline Other Hispanic & 1490 & $366(24.8)$ & $19.7,30.0$ & \\
\hline Other, incl multi-racial & 1018 & 107 (12.9) & $10.1,15.7$ & \\
\hline Poverty index ${ }^{\mathrm{d}, \mathrm{e}}$ & & & & $<0.0001$ \\
\hline Below & 5807 & $744(14.3)$ & $12.5,16.1$ & \\
\hline At or above & 15454 & $1621(10.9)$ & $9.9,11.8$ & \\
\hline Country of origin $^{d}$ & & & & $<0.0001$ \\
\hline United States & 18358 & $1401(8.9)$ & $7.9,9.9$ & \\
\hline Mexico & 2661 & $614(24.1)$ & $21.4,26.7$ & \\
\hline Elsewhere & 2005 & $627(29.2)$ & $25.7,32.7$ & \\
\hline
\end{tabular}

${ }^{\mathrm{a}}$ For Continuous NHANES 1999-2004: IgG $\geq 10 \mathrm{IU}=$ positive; IgG $<10 \mathrm{IU}=$ negative. For Continuous NHANES 2009-2010: IgG $\geq 33 \mathrm{IU} / \mathrm{mL}=\mathrm{positive;} \mathrm{IgG} \mathrm{<33} \mathrm{IU/mL} \mathrm{=}$ negative. ${ }^{\mathrm{b}} \mathrm{T}$. gondii serology performed only among participants ages 6-49 for 1999-2004 data. ${ }^{\mathrm{c} A l l}$ prevalence estimates were weighted. ${ }^{\mathrm{d} C o v a r i a t e}$ has some missing data. ${ }^{e}$ Family income-to-poverty ratio (FIPR) categorized as $0.00-0.99=$ below poverty; 1.00 and above $=$ at or above poverty.

with T. gondii seropositivity. To test this hypothesis, we next examined the association of drinking water and IgG antibodies against $T$. gondii separately among US-born and foreign-born participants (Table 3).

\section{US-born participants}

The final multivariable model included NHANES survey year, age, gender, household food security, race/ethnicity, smoking status, general health condition and source/treatment of tap water (Table 3). Mexican Americans and other Hispanics were less likely to be seropositive compared to non-Hispanic Whites $(\mathrm{AOR}=0.5 ; 95 \% \mathrm{CI}, 0.4-0.7$ and $\mathrm{AOR}=0.5$; 95\% CI, 0.3-0.98; respectively). Smokers had a higher odds of seropositivity compared to non-smokers $(\mathrm{AOR}=1.3$; 95\% CI, 1.1-1.6), and participants who reported fair or poor health were more likely to be seropositive (AOR $=1.5$; 95\% CI, 1.1-1.9) than participants who reported very good or good health, when compared to participants who reported excellent health. Participants with homes on well water (both those who used athome water treatment devices on the well water [AOR = 1.6; $95 \% \mathrm{CI}, 1.2-2.1]$ and those who did not $[\mathrm{AOR}=1.7$; 95\% CI, 1.1-2.4]), as well as participants using homeuntreated company tap water $(\mathrm{AOR}=1.2$; 95\% CI, 1.01.1.5) had significantly higher odds of seropositivity compared to participants using home-treated tap water provided by a private or public water company.

To expand on the comparative subpopulation analysis previously reported by Jones et al., among US-born persons 12-49 years old, T. gondii age-adjusted seroprevalence 
Table 2 Risk factors associated with antibodies ${ }^{\text {a }}$ against Toxoplasma gondii among participants $\geq 6$ years old; NHANES 1999-2004, 2009-10

\begin{tabular}{|c|c|c|c|c|}
\hline Variable & $\mathbf{N}$ & $\begin{array}{c}\text { IgG positive } \\
\text { n (\%) }\end{array}$ & $\begin{array}{c}\text { Unadjusted } \mathrm{OR}^{\mathrm{d}} \\
(95 \% \mathrm{Cl})\end{array}$ & $\begin{array}{c}\text { Adjusted } \mathrm{OR}^{\mathrm{d}} \\
(95 \% \mathrm{Cl})\end{array}$ \\
\hline \multicolumn{5}{|l|}{ Survey year } \\
\hline $1999-2000$ & 5089 & $602(13.3)$ & Ref & Ref \\
\hline $2001-2002$ & 5751 & $527(9.3)$ & $0.7(0.5-0.9)^{\mathrm{e}}$ & $0.7(0.5-0.9)$ \\
\hline 2003-2004 & 5120 & $434(10.8)$ & $0.8(0.6-1.0)$ & $0.8(0.6-1.1)$ \\
\hline $2009-2010$ & 7070 & 1079 (13.2) & $1.0(0.8-1.2)$ & $0.6(0.5-0.7)$ \\
\hline \multicolumn{5}{|l|}{ Age } \\
\hline Continuous (years) & 23030 & $2642(11.7)$ & $1.03(1.03-1.03)$ & $1.03(1.03-1.04)$ \\
\hline \multicolumn{5}{|l|}{ Age group (years) } \\
\hline $6-12$ & 4274 & $155(3.5)$ & Ref & - \\
\hline $13-17$ & 4693 & $279(4.6)$ & $1.4(1.03-1.8)$ & - \\
\hline $18-28$ & 4810 & $488(9.2)$ & $2.8(2.3-3.5)$ & - \\
\hline $29-43$ & 4799 & $757(14.0)$ & $4.5(3.6-5.8)$ & - \\
\hline$\geq 44$ & 4450 & $963(17.7)$ & $6.0(4.5-8.0)$ & - \\
\hline \multicolumn{5}{|l|}{ Gender } \\
\hline Female & 11791 & $1240(10.8)$ & Ref & Ref \\
\hline Male & 11239 & $1402(12.6)$ & $1.2(1.04-1.4)$ & $1.2(1.1-1.4)$ \\
\hline \multicolumn{5}{|l|}{ Race/Ethnicity } \\
\hline Non-Hispanic White & 8546 & 854 (9.9) & Ref & Ref \\
\hline Non-Hispanic Black & 5535 & $566(12.6)$ & $1.3(1.1-1.6)$ & $1.3(1.04-1.6)$ \\
\hline Mexican American & 6441 & $749(13.6)$ & $1.4(1.2-1.7)$ & $0.5(0.4-0.7)$ \\
\hline Other Hispanic & 1490 & $366(24.8)$ & $3.0(2.3-4.0)$ & $1.6(1.3-2.1)$ \\
\hline Other, incl. multiracial & 1018 & $107(12.9)$ & $1.3(1.02-1.8)$ & $0.7(0.5-1.0)$ \\
\hline \multicolumn{5}{|l|}{ Household food security ${ }^{f}$} \\
\hline Full food security & 15104 & $1593(10.9)$ & Ref & Ref \\
\hline Marginally secure or insecure & 7212 & $973(14.5)$ & $1.4(1.2-1.6)$ & $1.3(1.1-1.5)$ \\
\hline \multicolumn{5}{|l|}{ Smoking status, } \\
\hline Non-smoker & 18438 & $1977(11.0)$ & Ref & Ref \\
\hline Light smoker/moderate second-hand exposure & 1387 & $194(13.0)$ & $1.2(1.0-1.5)$ & $1.1(0.9-1.4)$ \\
\hline Smoker & 3045 & $447(14.1)$ & $1.3(1.1-1.6)$ & $1.2(1.03-1.5)$ \\
\hline \multicolumn{5}{|l|}{ Country of origin ${ }^{f}$} \\
\hline United States & 18358 & $1401(8.9)$ & Ref & Ref \\
\hline Mexico & 2661 & $614(24.1)$ & $3.2(2.7-3.9)$ & $6.3(4.7-8.4)$ \\
\hline Elsewhere & 2005 & $627(29.2)$ & $4.2(3.4-5.2)$ & $3.7(3.0-4.6)$ \\
\hline \multicolumn{5}{|l|}{ Source and home treatment ${ }^{\mathrm{h}}$ of tap water ${ }^{\mathrm{f}}$} \\
\hline Home-treated private/public water company & 4129 & $420(9.9)$ & Ref & Ref \\
\hline Home-untreated private/public water company & 16089 & $1855(12.1)$ & $1.2(1.1-1.4)$ & $1.2(1.003-1.4)$ \\
\hline Home-treated well water & 1056 & $143(12.7)$ & $1.3(1.05-1.7)$ & $1.4(1.1-1.8)$ \\
\hline Home-untreated well water & 1107 & $143(12.3)$ & $1.3(0.9-1.8)$ & $1.5(1.02-2.2)$ \\
\hline \multicolumn{5}{|l|}{ General health condition ${ }^{f}$} \\
\hline Excellent & 6341 & $477(8.5)$ & Ref & Ref \\
\hline Very good & 6306 & $588(9.8)$ & $1.2(1.0-1.4)$ & $1.1(0.9-1.3)$ \\
\hline Good & 7090 & $920(13.3)$ & $1.7(1.4-1.9)$ & $1.2(1.04-1.4)$ \\
\hline
\end{tabular}




\begin{tabular}{|c|c|c|c|c|}
\hline Fair & 2835 & $552(19.2)$ & $2.6(2.2-2.9)$ & $1.5(1.3-1.8)$ \\
\hline Poor & 447 & $103(25.1)$ & $3.6(2.6-5.0)$ & $1.9(1.4-2.7)$ \\
\hline \multicolumn{5}{|c|}{ Took treatment for anemia in past 3 months ${ }^{f}$} \\
\hline No & 22397 & $2526(11.5)$ & Ref & Ref \\
\hline Yes & 625 & $113(18.5)$ & $1.7(1.3-2.3)$ & $1.4(1.01-2.0)$ \\
\hline \multicolumn{5}{|l|}{ Blood eosinophils number ${ }^{f}$} \\
\hline Continuous (1000 cells/uL) & 22940 & $2629(11.7)$ & $1.4(1.02-1.9)$ & $1.3(1.0-1.7)$ \\
\hline
\end{tabular}

significantly fell to $6.6 \%(95 \% \mathrm{CL}, 5.2-8.0)(P<0.0001)$ for the 2009-10 NHANES cycle, compared to 14.1\% (95\% CL, 12.7-15.5) during the Third NHANES (1988-1994) and 9.0\% (95\% CL, 7.5-10.4) during the Continuous NHANES 1999-2004 [27] (data not shown).

\section{Foreign-born participants}

After adjusting for residential environment, the final multivariable model included NHANES survey year, age, gender, household food security, race/ethnicity, percentage of lifetime lived in the United States, smoking status, general health condition, source of tap water, anemia treatment, and eosinophilia (Table 3). In contrast to the US-born participants, among foreign-born participants, a higher $T$. gondii seroprevalence was observed among Hispanics other than Mexican American $(\mathrm{AOR}=2.3 ; 95 \% \mathrm{CI}, 1.7-3.0)$ and non-Hispanic Blacks $(\mathrm{AOR}=1.9 ; 95 \% \mathrm{CI}, 1.3-2.9)$. A lower seroprevalence was observed among "other" races, including multiracial ( $\mathrm{AOR}=0.5 ; 95 \% \mathrm{CI}, 0.4-0.8)$. When considering the percentage of one's lifetime spent living in the United States, a clear trend was observed, where the lower the percentage of lifetime, the higher the likelihood of $T$. gondii seropositivity $(\mathrm{AOR}=2.5 ; 95 \% \mathrm{CI}, 1.9-3.4$ for living $\leq 40 \%$ of lifetime compared to $\geq 76 \%$ ). In regards to health status, as observed in US-born participants, foreign-born participants who reported a fair or poor general health condition were more likely to be seropositive (AOR $=1.6 ; 95 \% \mathrm{CI}, 1.2-2.2$ ). In addition, participants with a blood eosinophil count above the normal range ( $\geq 3500$ cells/uL) and participants who reported taking treatment for anemia in the past 3 months were significantly more likely to be seropositive (AOR = 1.5; $95 \% \mathrm{CI}, 1.2-1.8$ and $\mathrm{AOR}=1.8$; $95 \% \mathrm{CI}, 1.1-3.0$; respectively). Source of tap water and using home water treatment devices were not associated with $T$. gondii seroprevalence among foreign-born participants.

\section{Discussion}

We observed statistically significant increased odds of $T$. gondii seropositivity among US-born NHANES participants whose tap water source was well water (both home treated and untreated), as well as home-untreated company water, compared to participants who used hometreated water from a private or public water company. The absence of significant associations between tap water source/treatment and $T$. gondii seropositivity among those not born in the United States provides additional evidence that drinking water may be an important source of toxoplasmosis in the United States.

Although $T$. gondii oocysts are resistant to chlorine and other types of disinfection [4], because of their relatively large size (10-12 $\mu \mathrm{m})$, they should be removed by standard municipal treatment consisting of coagulation, flocculation, and settling prior to filtration [29]. While outbreaks of $T$. gondii have only been attributed to municipal waters using unfiltered water systems (Brazil and Canada), outbreaks could also occur following a water treatment breakdown or operation error [30]. In addition, T. gondii oocysts have been found to be widespread in wells located on farms [31] and can survive for long periods in soil and water [24]. A study conducted among farms in Poland reported an association between well water consumption and toxoplasmosis IgG seropositivity [31]. Not only was a positive correlation observed between drinking well water and $T$. gondii seropositivity among persons living on the farms $(P<0.05)$, but $T$. gondii DNA was found significantly more frequently in water samples collected from windlassoperated wells $(37.5 \%)$ than water from deep wells with a pump (6.2\%) and from water supply systems (0\%). Well water, especially private or from a small shared source, is probably much less likely to be filtered adequately to remove $T$. gondii oocysts. Although we lacked much necessary detail to draw definitive conclusions, our results are consistent with an association between well water and $T$. gondii infection. 
Table 3 Risk factors associated with antibodies ${ }^{a}$ against Toxoplasma gondii among participants $\geq 6$ years old by country of birth; NHANES 1999-2004, 2009-10

\begin{tabular}{|c|c|c|c|c|c|c|c|c|}
\hline \multirow[t]{3}{*}{ Variable } & \multicolumn{4}{|c|}{ US-born } & \multicolumn{4}{|c|}{ Foreign-born } \\
\hline & \multirow[t]{2}{*}{$\mathbf{N}$} & $\begin{array}{c}\lg G \\
\text { positive }^{c}\end{array}$ & $\begin{array}{l}\text { Unadjusted } \\
\text { OR }^{\mathrm{d}}\end{array}$ & $\begin{array}{l}\text { Adjusted } \\
\mathrm{OR}^{\mathrm{d}}\end{array}$ & $\mathrm{N}$ & $\begin{array}{c}\lg G \\
\text { positive }^{c}\end{array}$ & $\begin{array}{l}\text { Unadjusted } \\
\mathrm{OR}^{\mathrm{d}}\end{array}$ & $\begin{array}{l}\text { Adjusted } \\
\mathrm{OR}^{\mathrm{d}}\end{array}$ \\
\hline & & n (\%) & $(95 \% \mathrm{Cl})$ & $(95 \% \mathrm{Cl})$ & & n (\%) & $(95 \% \mathrm{Cl})$ & $(95 \% \mathrm{Cl})$ \\
\hline \multicolumn{9}{|l|}{ Survey year } \\
\hline 1999-2000 & 4010 & $320(9.7)$ & Ref & Ref & 1078 & $282(32.3)$ & Ref & Ref \\
\hline $2001-2002$ & 4699 & $276(7.4)$ & $0.7(0.5-1.1)$ & $0.7(0.5-1.1)$ & 1052 & $251(21.8)$ & $0.6(0.4-0.9)^{\mathrm{e}}$ & $0.6(0.4-0.9)$ \\
\hline 2003-2004 & 4248 & $231(7.9)$ & $0.8(0.5-1.2)$ & $0.8(0.5-1.2)$ & 872 & $203(28.0)$ & $0.8(0.5-1.3)$ & $0.8(0.6-1.1)$ \\
\hline 2009-2010 & 5401 & $574(10.3)$ & $1.1(0.8-1.4)$ & $0.6(0.4-0.7)$ & 1664 & $505(27.6)$ & $0.8(0.6-1.2)$ & $0.7(0.5-0.9)$ \\
\hline \multicolumn{9}{|l|}{ Age } \\
\hline Continuous (years) & 18358 & $1401(8.9)$ & $1.03(1.03-1.04)$ & $1.04(1.03-1.04)$ & 4666 & $1241(27.5)$ & $1.02(1.01-1.02)$ & $1.03(1.02-1.04)$ \\
\hline \multicolumn{9}{|l|}{ Gender } \\
\hline Female & 9481 & $652(8.2)$ & Ref & Ref & 2307 & $588(26.7)$ & Ref & Ref \\
\hline Male & 8877 & 749 (9.6) & $1.2(1.002-1.4)$ & $1.2(1.05-1.5)$ & 2359 & $653(28.2)$ & $1.1(0.9-1.3)$ & $1.1(0.9-1.4)$ \\
\hline \multicolumn{9}{|l|}{ Race/Ethnicity } \\
\hline Non-Hispanic White & 8131 & $756(9.2)$ & Ref & Ref & 415 & $98(22.8)$ & Ref & Ref \\
\hline Non-Hispanic Black & 5104 & 419 (10.0) & $1.1(0.9-1.4)$ & $1.2(0.9-1.5)$ & 430 & $147(38.3)$ & $2.1(1.4-3.1)$ & $1.9(1.3-2.9)$ \\
\hline Mexican American & 3773 & $140(3.8)$ & $0.4(0.3-0.5)$ & $0.5(0.4-0.7)$ & 2666 & $609(23.9)$ & $1.1(0.8-1.4)$ & $0.9(0.7-1.2)$ \\
\hline Other Hispanic & 727 & $37(4.3)$ & $0.4(0.3-0.8)$ & $0.5(0.3-0.98)$ & 760 & $329(44.1)$ & $2.7(2.0-3.6)$ & $2.3(1.7-3.0)$ \\
\hline Other, incl. multiracial & 623 & $49(10.8)$ & $1.2(0.8-1.8)$ & $1.5(1.0-2.3)$ & 395 & $59(15.4)$ & $0.6(0.4-0.9)$ & $0.5(0.4-0.8)$ \\
\hline \multicolumn{9}{|l|}{ Household food security $^{f}$} \\
\hline Full food security & 12523 & $942(8.7)$ & Ref & Ref & 2581 & $651(25.0)$ & Ref & Ref \\
\hline Marginally secure or insecure & 5246 & $414(9.3)$ & $1.1(0.9-1.3)$ & $1.3(1.01-1.6)$ & 1962 & $559(33.1)$ & $1.5(1.2-1.8)$ & $1.2(1.0-1.5)$ \\
\hline \multicolumn{9}{|l|}{ Renting vs. owning home } \\
\hline Owned/being bought & 10861 & $877(9.3)$ & Ref & - & 1956 & $459(23.7)$ & Ref & Ref \\
\hline Rented/other arrangement & 7332 & $498(8.0)$ & $0.9(0.7-1.0)$ & - & 2666 & $769(30.7)$ & $1.4(1.2-1.7)$ & $1.3(1.1-1.6)$ \\
\hline \multicolumn{9}{|l|}{ Smoking status, ${ }^{f, g}$} \\
\hline Non-smoker & 14427 & $933(7.7)$ & Ref & Ref & 4005 & $1044(27.3)$ & Ref & - \\
\hline Light smoker $/ 2^{\text {nd }}$ hand exposure & 1082 & $105(9.3)$ & $1.2(0.9-1.6)$ & $1.2(0.9-1.6)$ & 305 & $89(29.4)$ & $1.1(0.8-1.6)$ & - \\
\hline Smoker & 2717 & $351(12.9)$ & $1.8(1.5-2.1)$ & $1.3(1.1-1.6)$ & 329 & $96(26.7)$ & $1.0(0.7-1.4)$ & - \\
\hline \multicolumn{9}{|l|}{ Water source ${ }^{f}$} \\
\hline Home-treated ${ }^{\mathrm{h}}$ water company & 3267 & $194(6.9)$ & Ref & Ref & 861 & $226(26.2)$ & Ref & Ref \\
\hline $\begin{array}{l}\text { Home-untreated water } \\
\text { company }\end{array}$ & 12608 & $922(8.7)$ & $1.3(1.05-1.6)$ & $1.2(1.01-1.5)$ & 3476 & $933(28.3)$ & $1.1(0.9-1.4)$ & $1.0(0.7-1.3)$ \\
\hline Home-treated well water & 990 & $131(12.4)$ & $1.9(1.4-2.6)$ & $1.6(1.2-2.1)$ & 66 & $12(19.4)$ & $0.7(0.3-1.8)$ & $0.5(0.2-1.0)$ \\
\hline Home-untreated well water & 1016 & 119 (12.0) & $1.8(1.3-2.7)$ & $1.7(1.1-2.4)$ & 91 & $24(19.8)$ & $0.7(0.4-1.3)$ & $0.8(0.4-1.4)$ \\
\hline \multicolumn{9}{|l|}{ General health condition $^{f}$} \\
\hline Excellent & 5456 & $293(6.8)$ & Ref & Ref & 885 & $184(21.8)$ & Ref & Ref \\
\hline Very good & 5372 & $365(8.0)$ & $1.2(1.0-1.5)$ & $1.0(0.8-1.3)$ & 932 & $223(24.2)$ & $1.2(0.9-1.5)$ & $1.1(0.8-1.5)$ \\
\hline Good & 5360 & 450 (9.8) & $1.5(1.2-1.9)$ & $1.1(0.9-1.4)$ & 1728 & $470(28.5)$ & $1.4(1.1-1.8)$ & $1.2(0.9-1.6)$ \\
\hline Fair/poor & 2164 & $292(14.9)$ & $2.4(1.9-3.0)$ & $1.5(1.1-1.9)$ & 1116 & $363(36.1)$ & $2.0(1.6-2.6)$ & $1.6(1.2-2.2)$ \\
\hline \multicolumn{9}{|l|}{$\begin{array}{l}\text { Took treatment for anemia in past } \\
3 \operatorname{mos}^{5}\end{array}$} \\
\hline No & 17859 & 1334 (8.7) & Ref & - & 4533 & $1192(27.3)$ & Ref & Ref \\
\hline Yes & 493 & 65 (14.8) & $1.8(1.3-2.6)$ & - & 131 & $48(40.4)$ & $1.8(1.1-3.1)$ & $1.8(1.1-3.0)$ \\
\hline
\end{tabular}




\begin{tabular}{|c|c|c|c|c|c|c|c|c|}
\hline \multicolumn{9}{|l|}{$\begin{array}{l}\text { Blood eosinophils number } \\
\text { (1000 cells/uLf }\end{array}$} \\
\hline Normal $(<0.35)$ & 15960 & $1239(8.9)$ & Ref & - & 4061 & $1040(26.4)$ & Ref & Ref \\
\hline Above normal ( $\geq 0.35)$ & 2441 & $158(8.6)$ & $1.0(0.8-1.2)$ & - & 573 & $192(35.1)$ & $1.5(1.2-1.9)$ & $1.5(1.2-1.8)$ \\
\hline \multicolumn{9}{|c|}{ Percentage of lifetime lived in US ${ }^{f}$} \\
\hline$\geq 0.76$ & - & - & - & - & 1147 & $206(18.3)$ & Ref & Ref \\
\hline $0.61-0.75$ & - & - & - & - & 1044 & $295(29.7)$ & $1.9(1.4-2.5)$ & $1.9(1.4-2.5)$ \\
\hline $0.41-0.60$ & - & - & - & - & 1149 & $337(29.5)$ & $1.9(1.4-2.4)$ & $2.2(1.6-2.8)$ \\
\hline$\leq 0.40$ & - & - & - & - & 1175 & $364(32.5)$ & $2.2(1.7-2.7)$ & $2.5(1.9-3.4)$ \\
\hline
\end{tabular}

${ }^{\mathrm{a}}$ For Continuous NHANES 1999-2004: IgG $\geq 10 \mathrm{IU}=$ positive; lgG <10 IU = negative. For Continuous NHANES 2009-2010: lgG $\geq 33 \mathrm{IU} / \mathrm{mL}=$ positive; lgG <33 IU/mL = negative. ${ }^{\mathrm{b}} \mathrm{T}$. gondii serology performed only among participants ages 6-49 for Continuous NHANES 1999-2004 data. ${ }^{\mathrm{C}}$ All prevalence estimates were weighted. ${ }^{\mathrm{d}}$ Wald Chi-square logistic regression used. ${ }^{\mathrm{e} B o l d e d}$ font denotes statistically significant data $(P<0.05) .{ }^{\mathrm{f}} \mathrm{Covariate}$ has some missing data. ${ }^{\mathrm{g} B a s e d}$ on serum cotinine level (ng/mL); nonsmoker $<10$, light smoker/second-hand exposure $10-99$, smoker $\geq 100$. ' $T y$ pes of home water treatment devices included: Brita ${ }^{\oplus}$ or other pitcher water filter, ceramic or charcoal filter, water softener, aerator, reverse osmosis.

Because the information collected on the type of water treatment was limited, it is difficult to interpret the effect of additional home treatment. Some treatments included in the question such as water softeners, would not have any effect on T. gondii oocysts, and even most water filters are simply designed to reduce taste and odor or reduce some chemicals such as lead and chlorine. Because oocysts are 10-12 $\mu \mathrm{m}$ in size [32], some standard Brita filters, such as faucet $(0.5$ micron $)$ and pitcher ( 1 micron) filters are expected to effectively remove oocysts, but other filters are only designed for taste and odor improvement and would have no effect. Moreover, the effectiveness of home treatments is impacted by maintenance and upkeep of the filter system. The use of additional treatment may also signal increased awareness and concern of water contamination and such participants may be more careful about general hygiene and the water they choose to drink.

Our analyses confirmed previous reports that those born outside the United States had higher odds for $T$. gondii seropositivity, supporting the evidence that other countries have a higher endemicity of human toxoplasmosis. We further demonstrated that the percentage of one's lifetime lived in the United States was inversely associated with $T$. gondii infection. Due to the NHANES classification of country of birth origin, we were unable to conduct meaningful analyses of which countries represent a higher risk of $T$. gondii infection. Seroprevalence varied among different race/ethnicity groups when stratified by country of origin, which is consistent with previous reports $[25,28]$. Stratification by country of origin also suggested blood eosinophils above the normal range and reported treatment for anemia in the past 3 months were associated with $T$. gondii seropositivity for foreign-born participants, but not for US-born participants. While these factors may simply be correlates of infection and not clinically associated with $T$. gondii infections, molecular and experimental infection studies have discovered that $T$. gondii genotypes vary in virulence, and these genetic lineages have evolved in different regions of the world [1]. Highly clonal genotypes II and III, the primary lineages in North America and Europe are considered to be avirulent in immunocompetent persons. In contrast, atypical genotypes resulting from frequent genetic exchanges and genetic drift have been associated with greater clinical severity. High genetic diversity among T. gondii isolates has been found in South America, Africa, and Asia. Because the clinical symptoms of anemia and inflammation were observed only among seropositive foreign-born NHANES participants, perhaps those born outside the United States acquired toxoplasmosis from more virulent strains. Following ingestion, $T$. gondii parasites invade the gastrointestinal wall, disseminate, and elicit a strong immune response that triggers significant ancillary pathologies; anemia and eosinophilia have been reported in clinically severe cases of toxoplasmosis [33-37].

While the Continuous NHANES collects a wealth of information for a national, representative sample of the United States population, we do recognize its limitations. The cross-sectional survey design prevents us from inferring causality. In addition, combining datasets across multiple survey years can be a limitation, depending on the covariates of interest. Questions and medical exams are not constant across the survey years: response options change, questions/medical tests are added, and questions/ medical tests are dropped. In addition, several questionnaires and medical exams are administered to only subgroups (e.g. adults $\geq 20$ years old). Because previous papers of $T$. gondii seroprevalence among NHANES participants analyzed subgroups that had additional NHANES data, e.g. education level and occupation among adults $\geq 20$ years old [26], our goal was to maintain the largest cohort as possible for consistency and increased power. 
Unfortunately, there was very limited data for other risk factors of interest, including the presence of $T$. gondii in drinking water sources, occupations with soil and water exposure, mental health status (e.g. depression and schizophrenia), and overall dietary behaviors (e.g. eating undercooked meat). In addition, as a health and nutrition survey, NHANES does not collect data regarding exposure to recreational waters, a key interest in environmental $T$. gondii transmission. Antibodies against $T$. gondii have been found in several species of marine mammals off of both US coastlines [23], suggesting that freshwater runoff is contaminating sea water [38]. This raises the question about the risk to humans from accidentally swallowing contaminated water during recreational exposure to rivers, lakes, and ocean waters [4]. Despite these limitations, this study provides risk factor data that can inform future environmental assessments and prospective human studies to better link cause and effect for water-associated toxoplasmosis.

\section{Conclusions}

Our findings are consistent with national trends observed in previous studies of $T$. gondii seroprevalence among NHANES participants. Seroprevalence is declining in the United States, with only $6.6 \%$ of the US-born population 12-49 years of age having serological evidence of $T$. gondii infection during 2009-10, compared to 9.0\% during 19992004 and $14.1 \%$ during $1988-1994$. We similarly observed differences in seropositivity by country of birth origin and race/ethnicity. There was also a significant association with less than excellent self-reported general health condition and seropositivity. Furthermore, this is the first paper to report associations between tap water source and $T$. gondii among US-born residents; although, a casual association cannot be demonstrated due to the cross-sectional design of NHANES. In addition, because standard T. gondii serological assays cannot differentiate environmentally-acquired oocyst infections from foodborne tissue cyst infections, recently developed oocyst antigen-specific assays, including the enzyme-linked immunoassay developed by Hill et al. [39], are valuable tools to more accurately assess the disease burden of environmental exposures to $T$. gondii. In addition, further investigations are warranted to examine how well water may be contaminated with oocysts, what role well systems may play in maintaining and spreading oocysts, and which home water treatment devices are effective in eliminating oocysts from tap water.

\section{Competing interests}

The authors declare that they have no competing interests.

\section{Authors' contributions}

All authors have substantially contributed to this work. WSK, RRC, and TJW performed the data processing and statistical analyses. All authors wrote, edited, and approved the final manuscript.

\section{Authors' information}

The views expressed in this paper are those of the authors and do not necessarily reflect the views or policies of the U.S. Environmental Protection Agency. Mention of trade names or commercial products does not constitute endorsement or recommendation for use.

\section{Acknowledgements}

We thank Dr. Alfred Dufour and Dr. Eric Villegas for their review and comments. The manuscript has been subjected to the U.S. EPA's peer review and has been approved for publication.

This project was supported in part by an appointment to the Internship/ Research Participation Program at the Office of Research and Development, U.S. Environmental Protection Agency, administered by the Oak Ridge Institute for Science and Education through an interagency agreement between the U.S. Department of Energy and EPA.

\section{Author details}

1Oak Ridge Institute for Science and Education, Oak Ridge, Tennessee, USA. Environmental Public Health Division, Office of Research and Development, US Environmental Protection Agency, 104 Mason Farm Road, Chapel Hill, North Carolina, USA.

Received: 11 March 2014 Accepted: 3 July 2014

Published: 10 July 2014

\section{References}

1. Robert-Gangneux F, Darde ML: Epidemiology of and diagnostic strategies for toxoplasmosis. Clinical Microbiology Reviews 2012, 25(2):264-296

2. Nelson KE, Williams CM: Infectious disease epidemiology. Third edition Burlington, MA: Jones \& Bartlett Learning; 2014.

3. National Center for Health Statistics: National health and nutrition examination survey data. Hyattsville, MD: U.S. Department of Health and Human Services Centers for Disease Control and Prevention; 2013.

4. Jones JL, Dubey JP: Waterborne toxoplasmosis-recent developments. Exp Parasitol 2010, 124(1):10-25.

5. Lass A, Pietkiewicz H, Modzelewska E, Dumetre A, Szostakowska B, Myjak P. Detection of Toxoplasma gondii oocysts in environmental soil samples using molecular methods. Eur J Clin Microbiol Infect Dis 2009, 28(6):599-605.

6. Hill D, Dubey JP: Toxoplasma gondii: transmission, diagnosis and prevention. Clin Microbiol Infect 2002, 8(10):634-640.

7. Webster JP, Lamberton PH, Donnelly CA, Torrey EF: Parasites as causative agents of human affective disorders? the impact of antipsychotic, mood-stabilizer and anti-parasite medication on Toxoplasma gondii's ability to alter host behaviour. Proc Biol Sci 2006, 273(1589):1023-1030.

8. Shepp DH, Hackman RC, Conley FK, Anderson JB, Meyers JD: Toxoplasma gondii reactivation identified by detection of parasitemia in tissue culture. Ann Intern Med 1985, 103(2):218-221.

9. Montoya JG, Liesenfeld O: Toxoplasmosis. Lancet 2004, 363(9425):1965-1976.

10. Fekadu A, Shibre T, Cleare AJ: Toxoplasmosis as a cause for behaviour disorders-overview of evidence and mechanisms. Folia Parasitol (Praha) 2010, 57(2):105-113.

11. Pearce BD, Kruszon-Moran D, Jones JL: The relationship between Toxoplasma gondii infection and mood disorders in the third National Health and Nutrition Survey. Biol Psychiatry 2012, 72(4):290-295.

12. Pappas G, Roussos N, Falagas ME: Toxoplasmosis snapshots: global status of Toxoplasma gondii seroprevalence and implications for pregnancy and congenital toxoplasmosis. Int J Parasitol 2009, 39(12):1385-1394.

13. Collier SA, Stockman L, Hicks LA, Garrison LE, Zhou FJ, Beach MJ: Direct healthcare costs of selected diseases primarily or partially transmitted by water. Epidemiol and Infect 2012, 140(11):2003-2013.

14. Cook AJ, Gilbert RE, Buffolano W, Zufferey J, Petersen E, Jenum PA, Foulon W, Semprini AE, Dunn DT: Sources of Toxoplasma infection in pregnant women: European multicentre case-control study: European research network on congenital toxoplasmosis. BMJ 2000, 321(7254):142-147.

15. Boyer KM, Holfels E, Roizen N, Swisher C, Mack D, Remington J, Withers S, Meier P, McLeod R, Toxoplasmosis Study Group: Risk factors for 
Toxoplasma gondii infection in mothers of infants with congenital toxoplasmosis: implications for prenatal management and screening. Am J Obstet Gynecol 2005, 192(2):564-571.

16. Tenter AM, Heckeroth AR, Weiss LM: Toxoplasma gondii: from animals to humans. Int J Parasitol 2000, 30(12-13):1217-1258.

17. Dubey JP: Toxoplasma gondii oocyst survival under defined temperatures. J Parasitol 1998, 84(4):862-865.

18. Dumetre A, Le Bras C, Baffet M, Meneceur P, Dubey JP, Derouin F, Duquet $J P$, Joyeux M, Moulin L: Effects of ozone and ultraviolet radiation treatments on the infectivity of Toxoplasma gondii oocysts. Vet Parasitol 2008, 153(3-4):209-213.

19. Ware MW, Augustine SA, Erisman DO, See MJ, Wymer L, Hayes SL, Dubey JP, Villegas EN: Determining UV inactivation of Toxoplasma gondii oocysts by using cell culture and a mouse bioassay. Appl Environ Microbiol 2010, 76(15):5140-5147.

20. Benenson MW, Takafuji ET, Lemon SM, Greenup RL, Sulzer AJ: Oocysttransmitted toxoplasmosis associated with ingestion of contaminated water. N Engl J Med 1982, 307(11):666-669.

21. Bowie WR, King AS, Werker DH, Isaac-Renton JL, Bell A, Eng SB, Marion SA: Outbreak of toxoplasmosis associated with municipal drinking water: the BC Toxoplasma investigation team. Lancet 1997, 350(9072):173-177.

22. Burnett AJ, Shortt SG, Isaac-Renton J, King A, Werker D, Bowie WR: Multiple cases of acquired toxoplasmosis retinitis presenting in an outbreak. Ophthalmology 1998, 105(6):1032-1037.

23. Dubey JP, Jones JL: Toxoplasma gondii infection in humans and animals in the United States. Int J Parasitol 2008, 38(11):1257-1278.

24. Torrey EF, Yolken RH: Toxoplasma oocysts as a public health problem. Trends Parasitol 2013, 29(8):380-384.

25. Jones JL, Kruszon-Moran D, Wilson M, McQuillan G, Navin T, McAuley JB: Toxoplasma gondii infection in the United States: seroprevalence and risk factors. Am J Epidemiol 2001, 154(4):357-365.

26. Jones JL, Kruszon-Moran D, Wilson M: Toxoplasma gondii infection in the United States, 1999-2000. Emerg Infect Dis 2003, 9(11):1371-1374.

27. Jones JL, Kruszon-Moran D, Sanders-Lewis K, Wilson M: Toxoplasma gondii infection in the United States, 1999 2004, decline from the prior decade. Am J Trop Med Hyg 2007, 77(3):405-410,

28. McQuillan GM, Kruszon-Moran D, Kottiri BJ, Curtin LR, Lucas JW, Kington RS: Racial and ethnic differences in the seroprevalence of 6 infectious diseases in the United States: data from NHANES III, 1988-1994. Am J Public Health 2004, 94(11):1952-1958.

29. Betancourt WQ, Rose JB: Drinking water treatment processes for removal of cryptosporidium and giardia. Vet Parasitol 2004, 126(1-2):219-234.

30. Craun GF, Brunkard JM, Yoder JS, Roberts VA, Carpenter J, Wade T, Calderon RL, Roberts JM, Beach MJ, Roy SL: Causes of outbreaks associated with drinking water in the United States from 1971 to 2006. Clin Microbiol Rev 2010, 23(3):507-528.

31. Sroka J, Wojcik-Fatla A, Dutkiewicz J: Occurrence of Toxoplasma gondii in water from wells located on farms. Ann Agric Environ Med 2006, 13(1):169-175.

32. Dubey JP, Lindsay DS, Speer CA: Structures of Toxoplasma gondii tachyzoites, bradyzoites, and sporozoites and biology and development of tissue cysts. Clin Microbiol Rev 1998, 11(2):267-299.

33. MacLaren A, De Souza W: Further studies on the interaction of Toxoplasma gondii with neutrophils and eosinophils. I Submicrosc Cytol Pathol 2002, 34(1):99-104.

34. Johnson LL, Berggren KN, Szaba FM, Chen W, Smiley ST: Fibrin-mediated protection against infection-stimulated immunopathology. J Exp Med 2003, 197(6):801-806.

35. Michelson AD, Lammi AT: Haemolytic anaemia associated with acquired toxoplasmosis. Aust Paediatr J 1984, 20(4):333-335

36. Neves ES, Bicudo LN, Curi AL, Carregal E, Bueno WF, Ferreira RG, Amendoeira MR, Benchimol E, Fernandes O: Acute acquired toxoplasmosis: clinical-laboratorial aspects and ophthalmologic evaluation in a cohort of immunocompetent patients. Mem Inst Oswaldo Cruz 2009, 104(2):393-396.

37. Nakazaki S, Saeki N, Itoh S, Osato K, Watanabe O, Hamada N, Mitsuhashi H, Shin H, Kiuchi I, Kobayashi C, Yano A, Yamaura A: Toxoplasmic encephalitis in patients with acquired immunodeficiency syndrome-four case reports. Neurol Med Chir (Tokyo) 2000, 40(2):120-123.

38. Conrad PA, Miller MA, Kreuder C, James ER, Mazet J, Dabritz H, Jessup DA, Gulland F, Grigg ME: Transmission of Toxoplasma: clues from the study of sea otters as sentinels of Toxoplasma gondii flow into the marine environment. Int J Parasitol 2005, 35(11-12):1155-1168.

39. Hill D, Coss C, Dubey JP, Wroblewski K, Sautter M, Hosten T, Munoz-Zanzi C, Mui E, Withers S, Boyer K, Hermes G, Coyne J, Jagdis F, Burnett A, McLeod P, Morton H, Robinson D, McLeod R: Identification of a sporozoite-specific antigen from Toxoplasma gondii. J Parasitol 2011, 97(2):328-337.

doi:10.1186/1471-2458-14-711

Cite this article as: Krueger et al:: Drinking water source and human Toxoplasma gondii infection in the United States: a cross-sectional analysis of NHANES data. BMC Public Health 2014 14:711.

\section{Submit your next manuscript to BioMed Central and take full advantage of:}

- Convenient online submission

- Thorough peer review

- No space constraints or color figure charges

- Immediate publication on acceptance

- Inclusion in PubMed, CAS, Scopus and Google Scholar

- Research which is freely available for redistribution 